\title{
Design of IoT based Smart Home System
}

\author{
Monish Chandradhara ${ }^{1}$, Ashlin George ${ }^{2}$, Mohammed Faraaz ${ }^{3}$, Aryan Saraf ${ }^{4}$ \\ ${ }^{1}$ Student, Dept of Mechanical Engineering, R.V. College of Engineering, Bangalore 560059 \\ ${ }^{2}$ Student, Dept of Mechanical Engineering, R.V. College of Engineering, Bangalore 560059 \\ ${ }^{3}$ Student, Dept of Mechanical Engineering, R.V. College of Engineering, Bangalore 560059 \\ ${ }^{4}$ Student, Dept of Mechanical Engineering, R.V. College of Engineering, Bangalore 560059

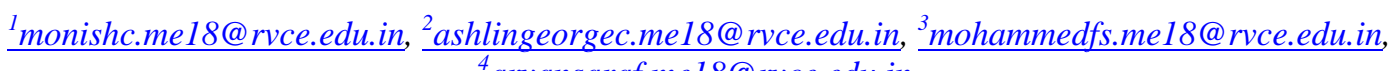 \\ aryansaraf.me18@rvce.edu.in.
}

\begin{abstract}
The Internet of Things (IoT) is used to describe a network of physical objects which are connected to each other with the help of embedded sensors, transceivers and software code to communicate and share data with each other. The Internet of Things, which first took shape back in the 1980s through an ARPA-NET connected Coca-Cola Vending machine in Carnegie Mellon University has come a long way since then.

In today's world a growing proportion of new home appliances are coming with IoT features embedded in them. These include, but are not limited to Television Sets, Refrigerators, Microwave Ovens, Fitness trackers, Smart watches and even IoT enabled light bulbs. IoT devices are not only used in Homes but also in Industries where they can play a pivotal role in bringing down operating costs and helping companies make better business decisions to align their companies in the right direction. With the advent of Industrial Internet of Things (IIoT) and Industry 4.0, a new standard has been set that organizations follow to gain a lot of key advantages such as improving productivity, customer experience, driving down costs and taking better informed decisions.
\end{abstract}

IoT devices have also entered the healthcare industry where they play a paramount role in monitoring vital signs of patients. These devices continuously monitor all the vital signs such as heart rate, blood pressure and oxygen saturation.

Keywords: Internet of Things, Arduino, Smart Home, HC-05 Bluetooth Module.

\section{1: Introduction}

\subsection{Market Survey}

The consumer IoT market is estimated to reach USD 104.4 billion by 2023 from USD 46.8 billion by 2018, at a CAGR of 17.39\% during 2018-2023. A few of the major driving factors for the growth of this market include the growing number of internet users and adoption of smart devices, increasing awareness about fitness and rise in disposable incomes in developing economies, and consumer preference for increased convenience and better lifestyle. Some of the key players in this segment driving the growth are large scale companies such as Qualcomm Incorporated (US), Texas Instruments Incorporated (US), NXP Semiconductors N.V. (Netherlands), Intel Corporation (US), STMicroelectronics N.V. (Switzerland), International Business Machines Corporation (IBM) 
(US), General Electric (GE) (US), Symantec Corporation (US), TE Connectivity Ltd. (Switzerland), Schneider Electric SE (France), Cisco Systems, Inc. (US), Amazon.com, Inc. (US), Apple Inc. (US), Alphabet Inc. (US), LG Electronics Inc. (South Korea), Samsung Electronics Co., Ltd. (South Korea).

\section{CONSUMER IOT MARKET, BY REGION (USD BILLION)}

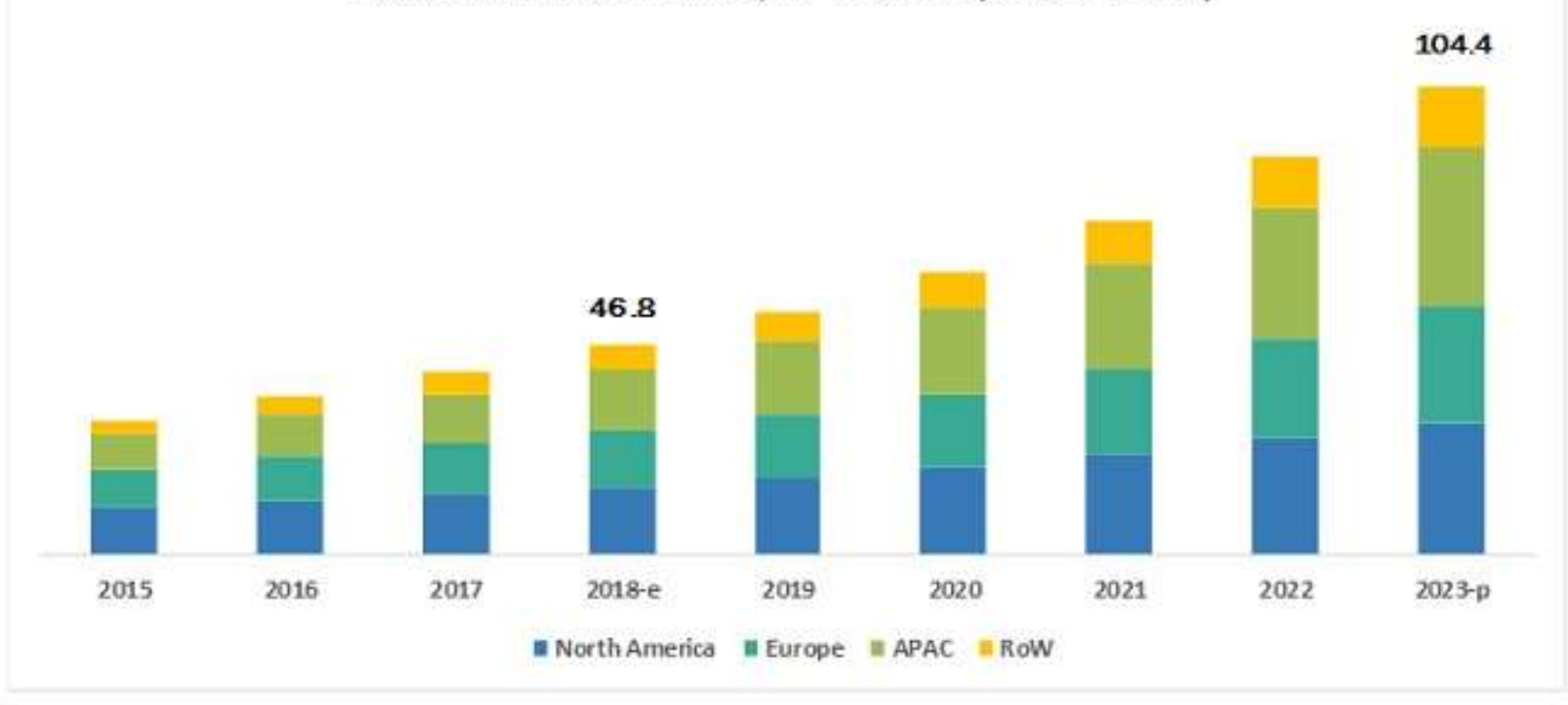

Note: $\mathrm{e}=$ Estimated Year and $\mathrm{p}=$ Projected Year

Figure 1.1

\subsection{Home Automation using Internet of Things}

Smart home automation is the practice of using internet enabled devices to remotely and automatically control appliances, in and around your home. You could use your phone to control most of these appliances. Smart homes are gaining popularity day-by-day with some numerous benefits. Industries and researchers are working hard to build sustainable and low-cost automatic systems to monitor and control different machines like lights, fans and motors. Smartphones can be used to control devices in a smart home. Systems based on the Bluetooth protocol are faster than most of the other technologies. Bluetooth is very effective for serial data communication where data can be transmitted at speeds of up to 3 mbps at a distance of I0 - $100 \mathrm{~m}$. Automation systems not only alleviate the wastage of electricity in a house but also reduce human effort in switching on and off home appliances. Home automation is an important application of wireless technologies like Bluetooth. Homes require sophisticated control of different gadgets such as electronic appliances. Home appliances can be integrated to smartphones via Bluetooth. Bluetooth works at a frequency range of $2.4 \mathrm{GHz}$ over a distance of $100 \mathrm{~m}$ with a speed of $1 \mathrm{Mbps}$. This is an efficient solution for controlling the process of home automation. A microcontroller can be used as a device controller for home devices. The significance of using the Bluetooth protocol for communication in a smart home stem from the fact that. Bluetooth is found in nearly every smartphone today. Hence having a smart home. where the microcontroller is Bluetooth enabled, makes it possible to easily control most devices with the aid of your 
smartphone which can save a lot of cost. Secondly most homes are not more than $200 \mathrm{~m}^{2}$ in floor area and Bluetooth protocol can cover a perimeter of a couple 100 meters hence this is the appropriate technology for this application.

\section{2 : Literature Review}

\subsubsection{Material and Network Protocol Selection}

Material selection was done based on the requirements and objectives of the smart home project and exploring all the different wireless technologies available to use to accomplish our objectives. The biggest decision to be made was the type of communication protocol to be adopted for the IoT system. The different types of network protocols available for IoT systems are:

- WiFi

- ZigBee

- Bluetooth

- Z-Wave

- LoRaWan

\section{WiFi}

WiFi uses radio waves that broadcast information on specific frequencies, such as $2.4 \mathrm{GHz}$ or $5 \mathrm{GHz}$ channels. Both frequency ranges have a number of channels through which different wireless devices can work, which helps to distribute the load so that the individual connections of the devices are not interrupted. This largely prevents overflowing of wireless networks. WiFi provides an Internet connection to nearby devices that are within a certain range. Another way to use WiFi is to create a WiFi hotspot, i.e. telephones or computers may share a wireless or wired internet connection with other devices by broadcasting a signal. A range of 100 meters is the typical range of a standard WiFi connection. The most common range, however, is limited to 10-35 meters. The effective network coverage is greatly affected by antenna strength or transmission frequency. The range and speed of a WiFi Internet connection depends on the environment and whether it provides internal or external coverage. Thus, the speed of various devices using the $\mathrm{WiFi}$ internet connection increases as the computer approaches the main source, while the speed decreases as the computer moves away from the source.

\section{ZigBee}

ZigBee-based networks are characterized by low power consumption, low throughputs (up to $250 \mathrm{kbps}$ ) and connectivity range of 100 meters between nodes. Typical applications include sensor networks, personal networks (WPAN), home automation, alarm systems and monitoring systems. Its initial specification was recognized as an IEEE standard in 2003 and the first OEM modules compliant with it ZigBee appeared in mass sales at the beginning 
of 2006. ZigBee was developed as a standard for self-configuring, short-range radio networks, intended for use in telemetry systems, for communication between various types of sensors, monitoring devices, as well as for wireless reading of measurement results from energy and heat meters, etc. The ZigBee standard is a relatively simple, resistant to communication errors and unauthorized readings, packet data exchange protocol, which is often implemented in devices with relatively small requirements, such as microcontrollers, sensors etc. ZigBee is easy to install and maintain because it is based on self-assembly and self-healing grid topology. It also easily scales to thousands of nodes, and nowadays there are many suppliers offering devices that support this open standard.

\section{Bluetooth}

Bluetooth is a technology that allows wireless connection of various electronic devices, such as a telephone, keyboard, computer, laptop, mouse, palmtop, printer, headset or speakerphone, and more. If you're down for a more wiki-like definition, this is an open standard described in the IEEE 802.15.1 specification and its technical specification includes three classes of ERP 1-3 transmission power with a range of, respectively, 100, 10 and 1 meter in open space. The most common class is the second one $(10 \mathrm{~m})$ which allows you to connect devices that are in different rooms and even on different floors. The standard uses radio waves in the $2.4 \mathrm{GHz}$ ISM frequency band and the device enabling the use of this standard is a Bluetooth adapter. In Bluetooth technology, data is sent in the form of packets to one of 79 channels (in the case of the oldest Bluetooth 1.0 standard) with a bandwidth of $1 \mathrm{Mhz}$ which ensures a maximum transfer speed of $721 \mathrm{kbit} / \mathrm{s}$. In the case of the latest Bluetooth 4.0 standard, there are 40 channels with a bandwidth of $2 \mathrm{Mhz}$, which guarantees a maximum data transfer of up to $3 \mathrm{Mb} / \mathrm{s}$. It is worth knowing that newer bluetooth standards that guarantee faster data transfer and greater security are also compatible with older versions.

\section{Z-Wave}

Z-Wave is an increasingly-popular IoT protocol. It's a wireless, radio frequency (RF) cased communication technology that's primarily used for IoT home applications. It operates on the $800-900 \mathrm{MHz}$ radio frequency. On the other hand, Zigbee operates on $2.4 \mathrm{GHz}$, which is also a major frequency for Wi-Fi. By operating in its own range, Z-Wave rarely suffers from any significant interference problems. However, the frequency that Z-Wave devices operate on is location dependent, so make sure you buy the right one for your country. Z-Wave is an impressive IoT protocol. However, like ZigBee, it's best used within the home and not within the business world.

\section{LoRaWan}

LoRaWAN is a media access control (MAC) IoT protocol. LoRaWAN allows low-powered devices to communicate directly with internet-connected applications over a long-range wireless connection. Moreover, it has the capability to be mapped to both the 2nd and 3rd layer of the OSI model. It's implemented on top of LoRa or FSK modulation for industrial, scientific, and medical (ISM) radio bands. 


\section{Component Selection :}

Based on our requirements and extensive research done on the different modules and microcontrollers available at our disposal, a calculated approach was taken to select the optimal components that are required to satisfy our objectives.

\section{Microcontroller - Arduino Uno - R3}

The Arduino Uno R3 is a microcontroller board based on a removable, dual-inline-package (DIP) ATmega328 AVR microcontroller. It has 20 digital input/output pins (of which 6 can be used as PWM outputs and 6 can be used as analog inputs). Programs can be loaded onto it from the easy-to-use Arduino computer program. The Arduino has an extensive support community, which makes it a very easy way to get started working with embedded electronics. The R3 is the third, and latest, revision of the Arduino Uno.

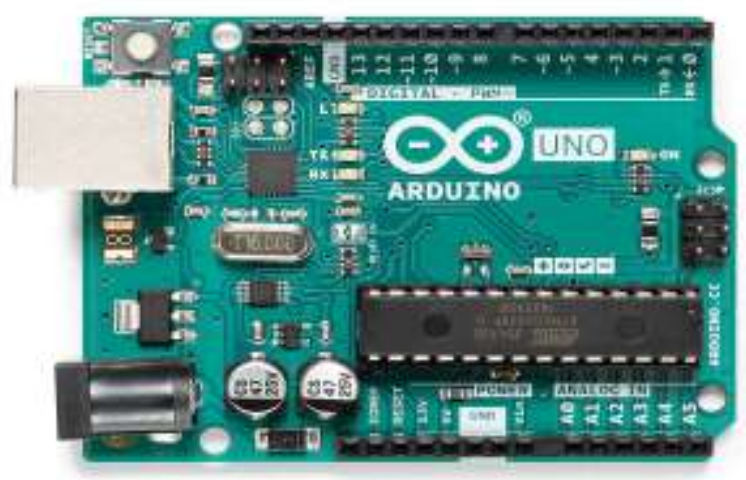

Figure 1.2: Picture of Arduino UNO R3 controller

The Arduino Uno is a microcontroller board based on the ATmega328. It has 20 digital input/output pins (of which 6 can be used as PWM outputs and 6 can be used as analog inputs), a $16 \mathrm{MHz}$ resonator, a USB connection, a power jack, an in-circuit system programming (ICSP) header, and a reset button. Based on our requirements of input voltage, number of input ports, availability of the microcontroller and the online support available for our module, we have decided to go with the Arduino Uno R3 controller. 


\section{Comparison of different Microcontrollers}

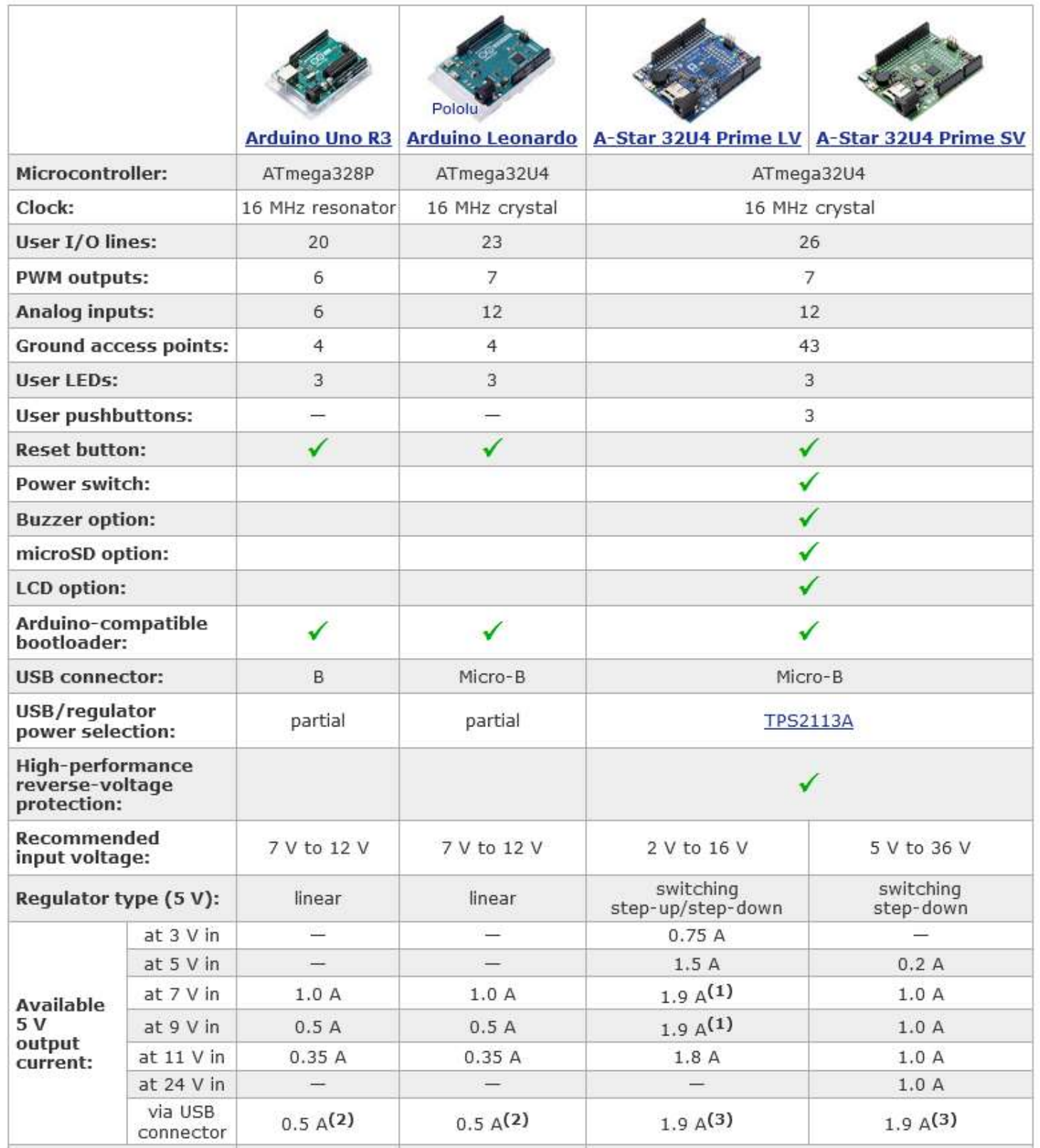

Table 1.1

\section{Connectivity Module - HCO5}

HC-05 Bluetooth Module is an easy to use Bluetooth SPP (Serial Port Protocol) module,designed for transparent wireless serial connection setup. Its communication is via serial communication which makes an easy way to interface with a controller or PC. HC-05 Bluetooth module provides switching mode 
between master and slave mode which means it is able to use neither receiving nor transmitting data. The input voltage into the module is $5 \mathrm{~V}$ and it is compatible with the Arduino Uno R3 as it's output is also $5 \mathrm{~V}$.

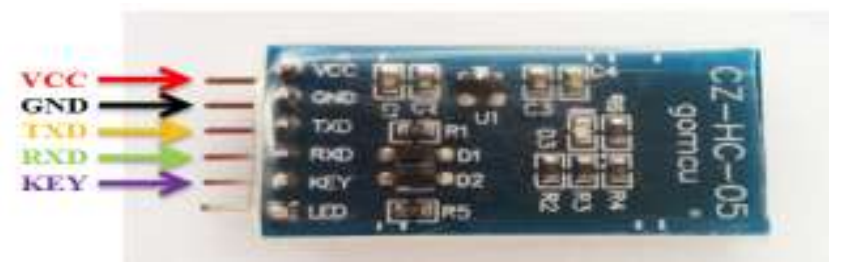

Figure 1.3: Picture of Bluetooth module (HC05)

\section{Pin Description for $\mathrm{HC} 05$}

\begin{tabular}{|l|l|l|}
\hline Pin & Description & Function \\
\hline VCC & $+5 \mathrm{~V}$ & Connect to $+5 \mathrm{~V}$ \\
\hline GND & Ground & Connect to Ground \\
\hline TXD & $\begin{array}{l}\text { UART_TXD, } \\
\text { Bluetooth serial } \\
\text { signal sending } \\
\text { PIN }\end{array}$ & $\begin{array}{l}\text { Connect with the MCU's (Microcontroller and etc) } \\
\text { RXD PIN. }\end{array}$ \\
\hline RXD & $\begin{array}{l}\text { UART_RXD, } \\
\text { Bluetooth serial } \\
\text { signal receiving } \\
\text { PIN }\end{array}$ & $\begin{array}{l}\text { Connect with the MCU's (Microcontroller and etc) } \\
\text { TXD PIN }\end{array}$ \\
\hline KEY & $\begin{array}{l}\text { Mode switch } \\
\text { input }\end{array}$ & $\begin{array}{l}\text { If it is input low level or connected to the air, the module is in } \\
\text { paired or communication mode. If it's input high level, the } \\
\text { module will enter AT mode. }\end{array}$ \\
\hline
\end{tabular}

Table 1.2

\subsection{Research Gap}

From the extensive literature survey done, certain safety issues and precautions to home automation systems were found. There are factors that can lead to device failure if the centralized communication hub stops working or breaks due to internal or external factors which would ultimately result in breakdown of the whole system. Therefore, proper backup measures including a self-unlock feature upon failure and a mechanical release can be provided in the central communication hub. The installation of this home automation system can lead to exposed security concerns, if not handled by professionals and efficient corporations who can invest and develop efficient cyber security. Invasion of privacy is a general concern if the smart parts manufactured by different companies are installed to 
check and control home security. Eavesdropping, compromised security concerns, hacking and intruding are some of the other concerns that can be avoided by taking strict security measures with cyber security practices.

It is also essential to design efficient home automation systems that have features that can reduce the power consumed by home appliances to improve the overall efficiency of the system.

\subsection{Main Objectives of the Project}

- To develop a mobile application-based home automation system to control home appliances

- To develop a prototype demonstrating the control of switching On/Off of lights as well as the intensity of the lights

- To develop a prototype demonstrating the control of fans

Methodology followed to complete the project included designing the circuit for the prototype on software platforms such as TinkerCAD and circuito.io, developing a small-scale prototype using an Arduino microcontroller, developing the code for the Arduino microcontroller to control the prototype, building the mobile application for user input using open source software platforms, and testing the final prototype.

\section{Chapter 3: Project Methodology}

\subsection{Problem Definition}

Usage of IoT applications to develop smart home systems has been a popular field of research in recent years, and the extent of its application could go on increasing. It is necessary to incorporate as many features as possible to develop a system that can automate and control all the appliances in the house but it is also essential to design efficient systems to reduce usage of electricity, hence adding features such as controlling the intensity of lighting can reduce the amount of power consumption by such appliances.

\subsection{Project Methodology}

All the individual components were selected before the circuit diagram was developed. LEDs were selected as light sources since one of the objectives of the project was to demonstrate the control of intensity of lights. Since connecting an LED directly with a voltage source could destroy the LED by causing it to burn out, 100 ohms resistors were connected in series to prevent the LED from getting damaged. In case of control of fan, the motor used had a voltage rating of $9 \mathrm{~V}$, but the Arduino output voltage is $5 \mathrm{~V}$, so instead of connecting the fan directly to the Arduino board, an external circuit was made for the fan which had a $9 \mathrm{~V}$ battery, and a relay was connected in series with the fan and the battery, and this relay was connected to the Arduino board. This relay gets energized when it receives an input current from the Arduino and closes the external circuit, thereby causing the fan to rotate. The 
relay gets de-energized when it does not receive a voltage signal and opens the external circuit, causing the fan to come to a stop.

Taking the above points into consideration, the circuit diagram schematic was designed. The first iteration of the circuit diagram was done on an online open source software platform called Tinker CAD by Autodesk. The limitation here was that the Bluetooth module required ( $\mathrm{HC} 05)$ was not available in the in-built library of components. So, another online open source software platform called cicuito.io was used.

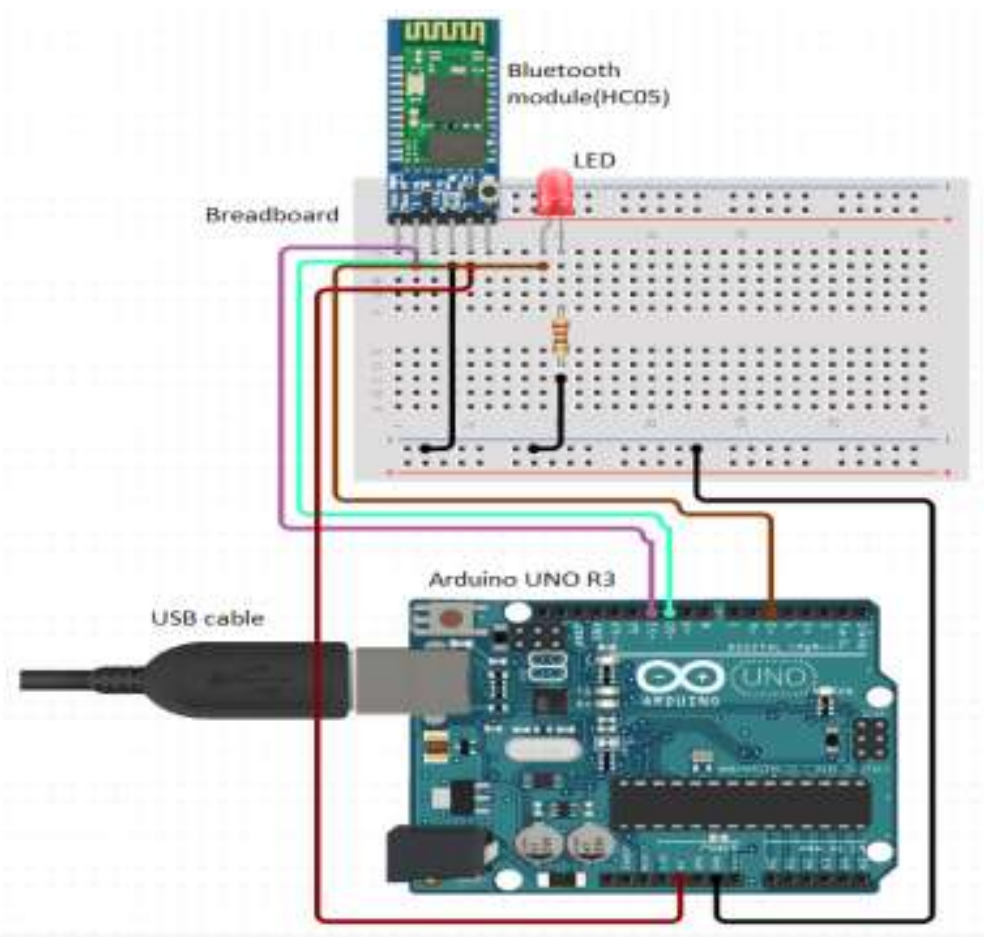

Figure 2.1: Circuit diagram for control of light 


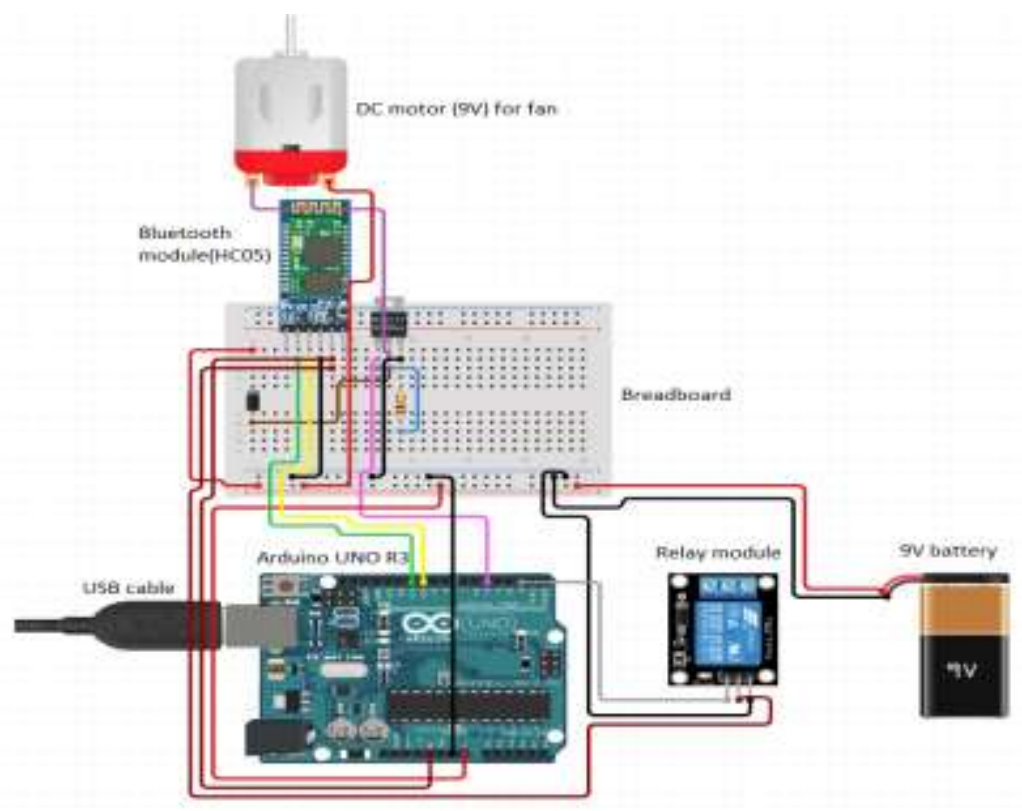

Figure 2.2: Circuit diagram for control of fan

The code to control the Arduino board was developed on the IDE for the Arduino. For building the mobile application where users provide their input, an online open-source android app platform that was compatible with the Bluetooth module was used and the control logic could be easily implemented in the Arduino code IDE. It had been used before to design IoT based mobile applications, hence it was a reliable option.

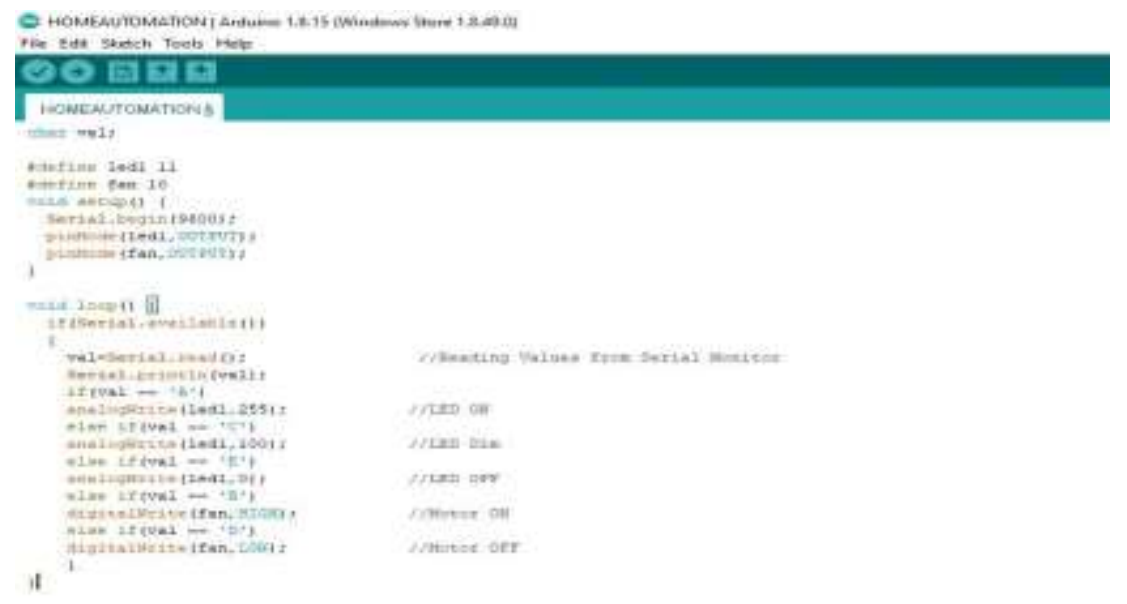

Figure 2.3: Screenshot of Arduino IDE with the code for control logic

\section{Chapter 4: Results and Discussion}

The code for logic implementation was developed and the circuit wiring was done. 


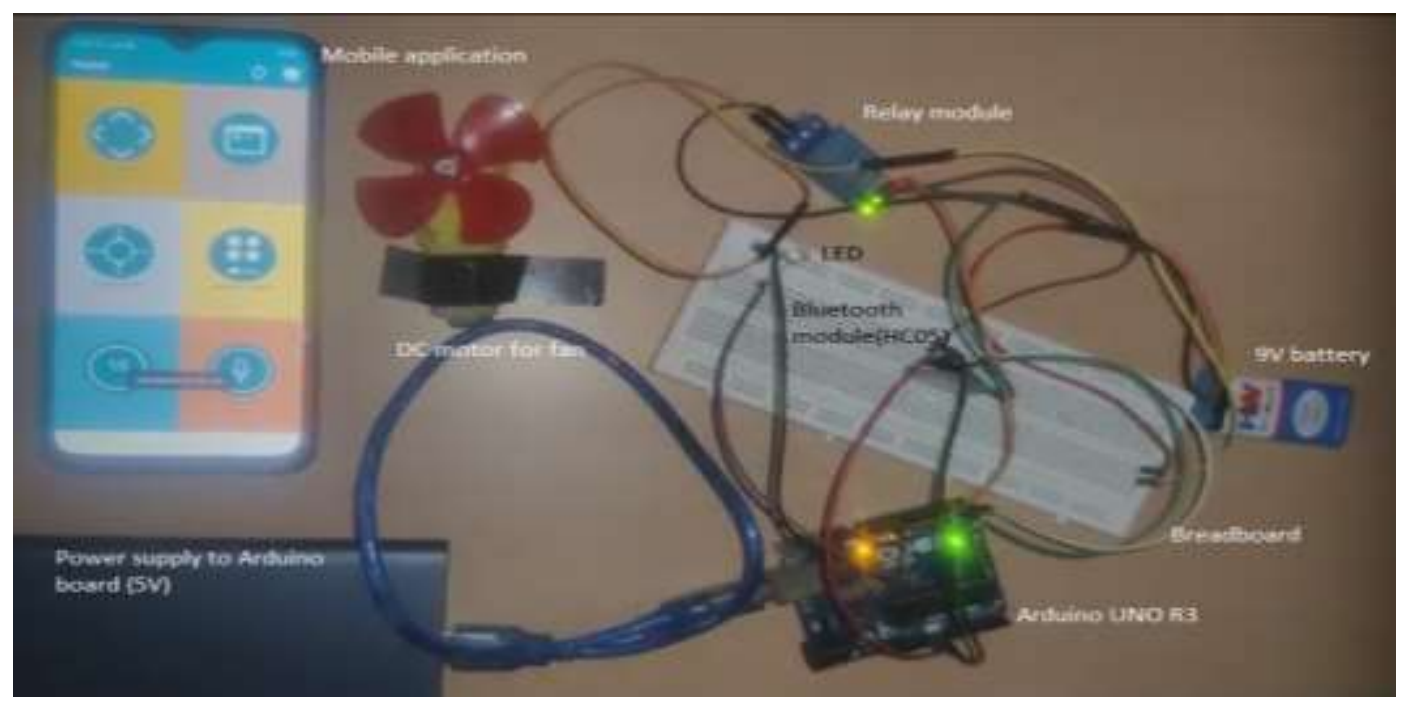

Figure 3.1: Circuit connection for the prototype

\subsection{Control of Lights}

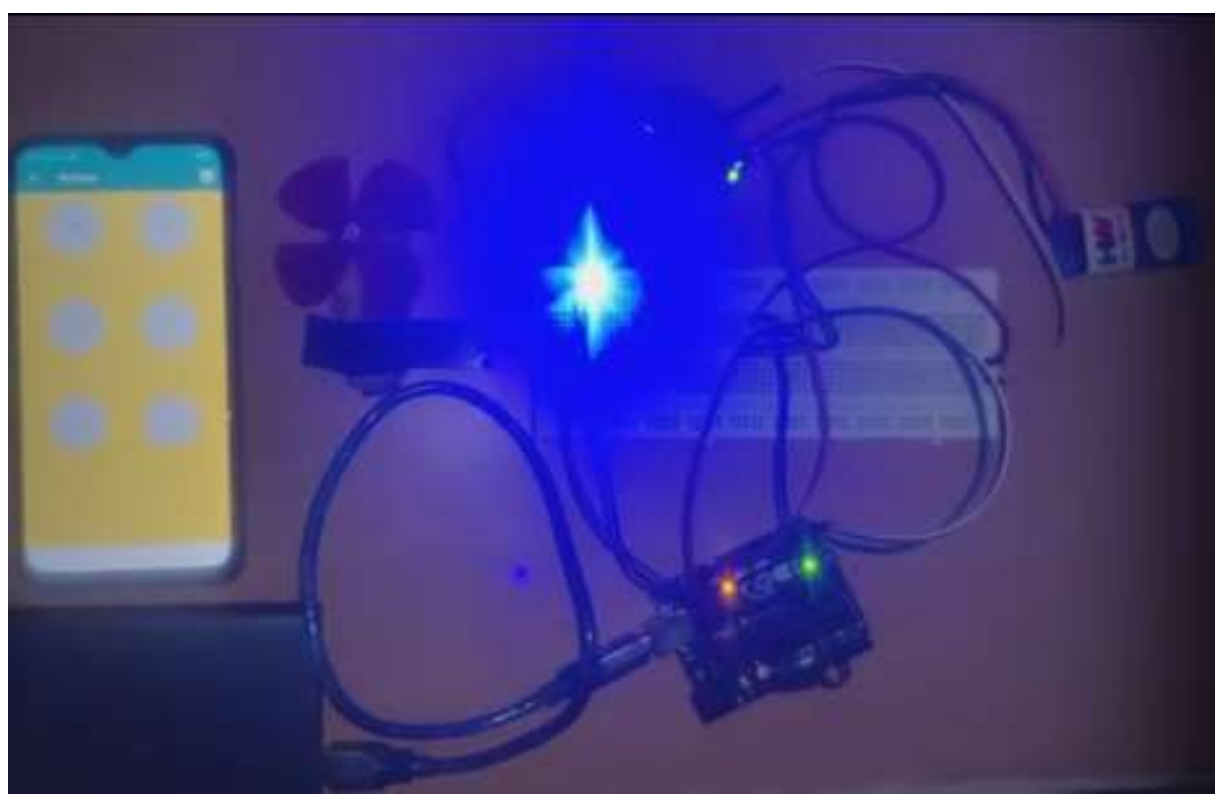

Figure 3.2: LED on at full intensity

The LED was connected across the analog pins of the Arduino board in order to provide varying voltage input to it to change the intensity of lighting. An output voltage of $5 \mathrm{~V}$ was used for the LED to glow at full intensity and an output voltage of $2 \mathrm{~V}$ was used to make it glow at an intermediate intensity. The switching between these voltages happens when the user provides input using the mobile application indicating whether full intensity or medium intensity is required, and the programmed control logic processes this input and an appropriate voltage signal is sent to the LED. 


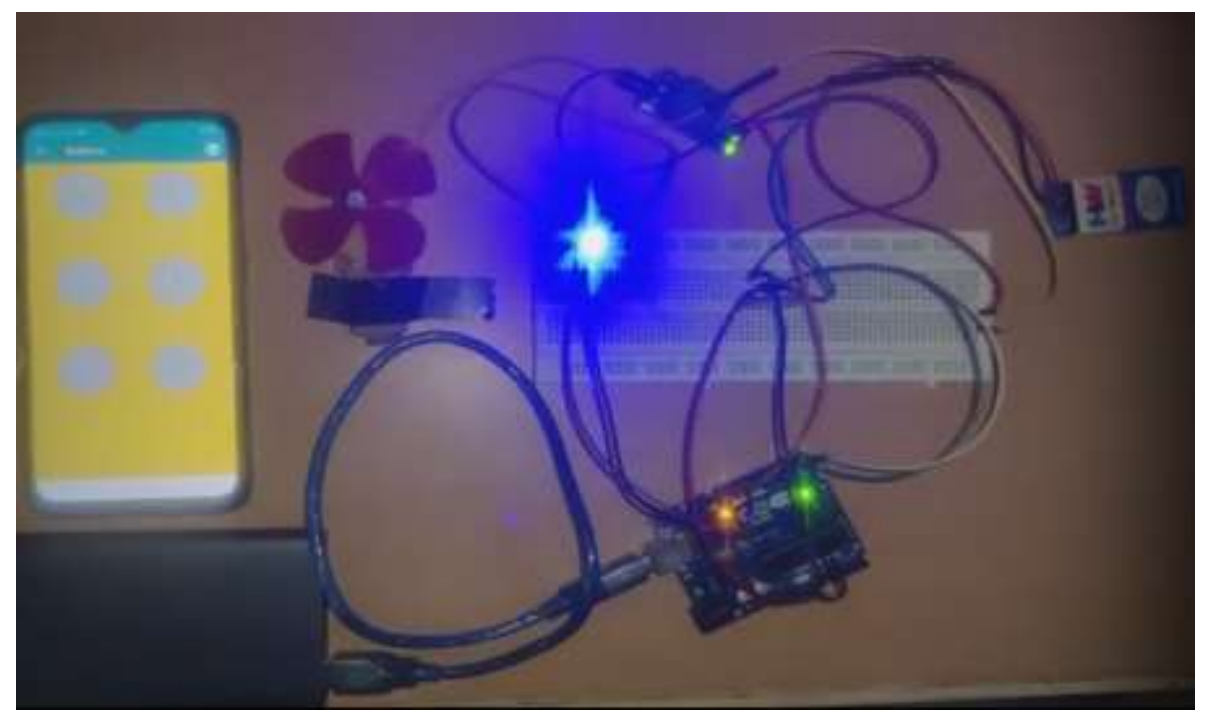

Figure 3.3: LED on at intermediate intensity

\subsection{Control of Fan}

A 9V DC motor was used for the fan. Since the Arduino output voltage is only 5V, instead of connecting the motor directly to the Arduino, an external circuit was made consisting of the $9 \mathrm{~V}$ battery and the motor, and a relay module was connected in series in this external circuit. This relay module was connected to the Arduino output. The relay works on the principle of electromagnetism and consists of a coil of wire that gets energized and acts as a solenoid when it receives the input current from the Arduino. When the relay coil is energized, the magnetic flux developed induces a force that causes the physical contacts to close thereby, closing the circuit and causing the fan to rotate. When there is no output voltage signal from the Arduino, the relay coil gets de-energized and the physical contacts are no longer in contact, thereby opening the circuit and causing the fan to come to rest.

\section{Chapter 4: Conclusions}

Usage of IoT applications to develop smart home systems has been a popular field of research in recent years, and the extent of its application could go on increasing. It is necessary to incorporate as many features as possible to develop a system that can automate and control all the appliances in the house but it is also essential to design efficient systems to reduce usage of electricity, hence adding features such as controlling the intensity of lighting can reduce the amount of power consumption by such appliances. In this project, a prototype to control some of these home appliances was developed, these features were tested and demonstrated successfully. 


\section{ACKNOWLEDGEMENT}

The successful completion of this project work was made possible through the valuable contribution of a number of people. To say thank you to all of them is not even enough to express our gratitude.

Our first debt of gratitude and deep regards must go to our guide Dr. Sridhar R, Associate Professor, Department of Mechanical Engineering for his valuable and inspiring guidance, wholehearted support, suggestions, patience and constant encouragement throughout our project work and his immense help in the preparation of this paper. The blessing, help and guidance given by them from time to time shall carry us a long way through our journey. Lastly, our special thanks go to our parents for their blessings have been a great source of inspiration for us.

\subsection{References}

[1]Rahabul Islam, Md. Wahidur Rahman, Rahmina Rubaiat, Md. Mahmodul Hasan, Md. Mahfuz Reza, Mohammad Motiur Rahman: LoRa and server-based home automation using the internet of things (IoT): 2020 [2]Rajkumar N, A B Rajendra, Prafulla, Viji Vinod: H2M communication for Home Appliances Automation using Android Application: ICCIDS: 2019

[3]S.R. Paveethra, B. Barathi, M. Geethapriya, M. Arthi, V. Ahasthiya: Theoretical modelling and implementation of home energy management system using IoT based automation system: Materials Today: Proceedings: 2020

[4]Kabita Agarwal, Arun Agarwal, Gourav Misra: Review and Performance Analysis on Wireless Smart Home and Home Automation using IoT: I-SMAC: 2019

[5]Gaurav Panwar, Rajat Maurya, Rajesh Rawat, Rohit Kanswal, Praful Ranjan: Home Automation using IOT Application: IJSH: 2017

[6]Jose Ripper Kos, Marcelo Contatto, James Miyamoto: Nature as an extended interface to home automation systems: IREE: 2017 